\title{
GOBERNANZA DE LOS RECURSOS HIDRICOS TRANSFRONTERIZOS: UNA PROPUESTA
}

\section{GOVERNANCE OF TRANSBOUNDARY WATER RESOURCES: A PROPOSAL}

\author{
Julián Mora Aliseda ${ }^{1}$ \\ Jacinto Garrido Velarde ${ }^{2}$ \\ Consuelo Mora Aliseda ${ }^{3}$.
}

\section{Resumen}

Existen numerosos precedentes de aplicación de la planificación estratégica y de los sistemas de planificación integrada a distintos ámbitos de la gestión de los recursos naturales. El antecedente más claro y evidente es la Estrategia Territorial Europea, consistente en un ambicioso proyecto y esfuerzo de homogeneización terminológica y de convención técnica en materia de ordenación territorial y urbana en el que se han involucrado todos los países de la Unión Europea. El modelo de la Estrategia Territorial Europea es sin duda un modelo muy válido, tanto como metodología de trabajo como de sistema de concertación de la clase investigadora y la clase política de los países implicados. En lo que toca específicamente a la planificación estratégica de los recursos hídricos y de la gestión del agua, evidentemente las experiencias existentes son escasas. Debe tenerse presente que hasta la fecha la gestión de los recursos hídricos y de las cuencas hidrográficas ha tenido un carácter muy sectorial y ha estado muy restringida al ámbito de actuación de las respectivas instituciones gubernamentales responsables de la gestión, esto es, las Confederaciones Hidrográficas en el caso español y el Instituto Nacional del Agua (INAG) en el caso portugués. Por ello, el presente estudio realiza un análisis de la Estrategia Territorial Europea como punto de partida para establecer la cooperación transfronteriza Hispano-

\footnotetext{
${ }^{1}$ Prof. Catedrático de la Universidad de Extremadura. Doctorado en Ordenación del Territorio y Doctorado en Sociología. Correo electrónico .tajoguadiana@gmail.com

2 Prof. Ayudante de la Univ. de Extremadura. Doctorado en Ingeniería. Másteres en SIG y Urbanismo. Correo electrónico igarridoif@gmail.com

${ }^{3}$ Fundicotex. Doctorada Geografía y Master en Antropología. Diplomada en Medio Ambiente y Desarrollo Local. Correo electrónico cmora63@gmail.com
} 
Lusa en la gestión de los recursos hídricos, estableciendo tras dicho análisis las propuestas metodológicas a seguir para abordar y concretar dicha cooperación fronteriza transnacional, en pro de la gestión de los recursos hídricos de la Península Ibérica.

Palabras Claves: Ordenación Territorial, Planificación Estratégica, Recursos Hídricos, Cooperación Transfronteriza

\section{Abstract}

There are numerous precedents for the application of strategic planning and integrated planning systems to different areas of natural resource management. The clearest and most obvious antecedent is the European Territorial Strategy, consisting of an ambitious project and effort to homogenize terminology and a technical convention on territorial and urban planning, in which all the countries of the European Union have been involved. The model of the European Territorial Strategy is undoubtedly a very valid model, both as a methodology of work and as a system of consultation between the research class and the political class of the countries involved. With regard to the strategic planning of water resources and water management, evidently existing experiences are scarce. It must be borne in mind that to date the management of water resources and watersheds has had a very sectoral nature and has been very restricted to the scope of action of the respective government institutions responsible for the management, that is, the Hydrographic Confederations in the Spanish case and the National Water Institute (NWI) in the Portuguese case. For this reason, this study analyzes the European Territorial Strategy as a starting point to establish cross-border cooperation between Spanish and Portuguese in the management of water resources, establishing after this analysis the methodological proposals to be followed to address and specify such transnational border cooperation, in favor of the management of the water resources of the Iberian Peninsula.

Keywords: Territorial Planning, Strategic Planning, Water Resources, Cross-Border Cooperation

\section{LA ESTRATEGIA TERRITORIAL EUROPEA}

En el año 1999 surge la Estrategia Territorial Europea (ETE), con el objetivo de progresar hacia la cohesión económica y social, la conservación de los recursos naturales y del patrimonio cultural y, la competitividad más equilibrada del territorio europeo. Ello sólo se podrá conseguir mediante la colaboración y coordinación de los 
diferentes niveles de gobierno y administración: gobiernos locales, regionales, nacionales y comunitarios. Para ello es necesario desarrollar un sistema equilibrado y policéntrico de ciudades y una nueva relación entre campo y ciudad; la garantía de un acceso equivalente a las infraestructuras y al conocimiento; y el desarrollo sostenible, la gestión inteligente y la protección de la naturaleza y del patrimonio cultural. No obstante, hay que llevar a cabo una buena planificación, pues una política centrada únicamente en el equilibrio provocaría el debilitamiento de las ciudades económicamente más fuertes, aumentando la dependencia de las ciudades más atrasadas. Pero si los esfuerzos comunitarios se centran en el desarrollo por sí solo provocaría el aumento de los desequilibrios ya existentes. Además una excesiva conservación de las estructuras territoriales aumentaría a su vez el riesgo de estancamiento, ya que las tendencias modernizadoras podrían verse paralizadas. Por tanto, mediante la combinación de los objetivos de desarrollo, equilibrio y conservación, junto con el raciocinio según las diferentes situaciones territoriales, será posible un desarrollo equilibrado y sostenible.

La ETE define que el desarrollo de los territorios europeos debe pasar por el desarrollo de un sistema urbano policéntrico y más equilibrado, junto con el esfuerzo de la colaboración entre los espacios urbanos y rurales; el fomento de estrategias integradas de transporte y comunicación que sirvan de ayuda para el desarrollo policéntrico del territorio comunitario, consiguiendo el acceso a infraestructuras y al conocimiento; y el desarrollo y protección de la naturaleza y del patrimonio cultural mediante una gestión inteligente. El modelo policéntrico -que debe contribuir a evitar que continúe concentrándose excesivamente la riqueza y la población en el núcleo central de la UE- debe consolidar una estructura territorial relativamente descentralizada. Hasta ahora las medidas desarrolladas se han centrado en la mejora de las conexiones entre la periferia y el núcleo central mediante proyectos de infraestructuras. Sin embargo, es necesaria una política que ofrezca nuevas perspectivas para las zonas periféricas mediante una conformación más policéntrica 
del espacio de la Comunidad. Por ello, el fomento de la complementariedad entre ciudades y regiones significa aprovechar las ventajas de la competencia económica entre ellas, que debe ampliarse a todas las funciones de la ciudad, siguiendo una política de fomento de la colaboración efectiva entre las ciudades basándose en intereses comunes y en la aportación de todos los participantes. Por ello hay que potenciar la cooperación en el transporte de cercanías, la definición de zonas residenciales o industriales comunes, o la gestión de residuos. Es asimismo importante la creación de redes de ciudades pequeñas en las regiones con menor densidad de población y económicamente más atrasadas, para que la combinación de estos potenciales urbanos pueda contribuir a alcanzar un número de consumidores suficientes para el mantenimiento de las empresas y servicios, que las distintas ciudades no podrían conseguir por sí solos.

El gran problema de las regiones más atrasadas de la UE es que sus ciudades se basan en una economía estrecha y dominada por una sola rama de actividad, cuyo declive tiene repercusiones negativas sobre la economía de toda la región. La competitividad de estas ciudades depende de una política de diversificación de su base económica y de la competitividad de sus ciudades. Para ello hay que potenciar el control de la expansión urbana; la mezcla de funciones y grupos sociales; la gestión inteligente y economizadora de los recursos del ecosistema urbano; la mejora de la accesibilidad gracias a medios de transporte compatibles con el medio ambiente y la protección y el desarrollo del patrimonio natural y cultural mediante una buena planificación estratégica.

El impulso de las regiones rurales pasa por potenciar su desarrollo endógeno. Algunos espacios rurales han conseguido superar con éxito un cambio estructural, lo que no está vinculado sólo a los factores de localización, como una ubicación atractiva o un nivel salarial más bajo, sino también a factores de patrimonio natural y cultural, a la presencia de redes y asociaciones, al carácter democrático de la toma de decisiones, y a la iniciativa y el compromiso de los políticos regionales y locales, así como de otros 
agentes sociales. Por eso, hay que buscar, mediante una planificación, la diversificación de la economía de estas zonas rurales para evitar una excesiva dependencia respecto a estructuras unisectoriales, y crear oportunidades de empleo estable.

Muchas ciudades europeas se exponen a los peligros de la comercialización y la uniformidad cultural, que destruye su individualidad y su identidad. Entre estos peligros se cuentan la especulación inmobiliaria, los proyectos de infraestructuras sobredimensionados en relación con el entorno, o unas adaptaciones poco meditadas a las exigencias del turismo de masas. Raramente se construyen edificios o conjuntos de edificios según una visión contemporánea del urbanismo y se insertan armoniosamente en el conjunto urbano. Al igual que en el medio rural, los paisajes urbanos son a menudo producto de una evolución aleatoria.

La aplicación de estas políticas se basa en el principio de subsidiariedad, por lo que es necesaria una cooperación de las políticas sectoriales entre sí y con las autoridades competentes de ordenación del territorio (cooperación horizontal), por una parte, y entre las políticas a escala comunitaria, transnacional, regional y local por otra (cooperación vertical).

Una política de planificación territorial integrada a escala de la UE debe combinar las opciones políticas destinadas a desarrollar determinadas zonas, de modo que las fronteras nacionales y otras dificultades administrativas dejen de representar obstáculos al desarrollo. Por ello, la ETE exalta tres niveles para la cooperación territorial: nivel comunitario, nivel transnacional/nacional y nivel regional/local. La ETE deriva una serie de tareas clave para una política de desarrollo equilibrado y sostenible como lo son: la integración en redes de las regiones urbanas, todas las ciudades y regiones deben estar en condiciones de contribuir a la reducción de la tasa de desempleo, al crecimiento económico y al equilibrio social en la UE; una mejor accesibilidad como condición previa de un desarrollo policéntrico; la creación de euro- corredores, que puedan reforzar la cohesión territorial de la UE y representen un instrumento esencial del desarrollo territorial para la cooperación de las ciudades; el refuerzo de las ciudades 
y regiones situadas junto a las fronteras exteriores de la UE; la conservación y desarrollo de la biodiversidad en las regiones de la UE, mediante la creación de una red europea de espacios naturales que requiere la coordinación territorial de las distintas políticas comunitarias entre sí y con las correspondientes actuaciones nacionales; desarrollo del patrimonio cultural europeo; gestión integrada de las zonas costeras, debido a la progresión de los conflictos sectoriales, a la evolución demográfica y al número de instituciones y agentes con intereses en las zonas costeras.

Para una mejor aplicación de las políticas que la ETE recomienda es necesario evaluar de manera sistemática y periódica a nivel europeo, nacional y local los efectos territoriales que las políticas comunitarias tienen.

La formación de redes de ciudades en el futuro vendrá ligada por la aparición de nuevas funciones y nuevas redes influirán en el futuro sobre la evolución de algunas ciudades y regiones. Las ciudades cooperan cada vez de forma más estrecha y unen sus fuerzas, por ejemplo, desarrollando funciones complementarias 0 aprovechando conjuntamente distintas instalaciones y servicios, tal cooperación puede favorecer el desarrollo regional, porque mejora la oferta de servicios y las condiciones económicas de la región, aumentando así su competitividad. Los factores que hacen muchas veces que esta cooperación sea turbulenta son la existencia de sistemas políticos y administrativos diferentes o las distancias entre ciudades en las regiones poco pobladas.

La evolución de las perspectivas económicas de las ciudades y regiones industriales debe continuar por su modernización económica. Las ciudades y regiones que dependen principalmente de una única actividad económica, por ejemplo, la administración pública, el turismo o las funciones portuarias, deberán intentar diversificar su base económica, mediante la planificación. Con todo, también en las regiones periféricas hay ciudades con potencial y atractivo suficiente para atraer inversiones hacia sí y su entorno. En particular, las ciudades que desempeñan la 
función de "ciudades-puerta" pueden explotar su emplazamiento periférico de manera muy favorable.

El crecimiento anárquico y sin planificar genera un desarrollo más intenso del tráfico privado, aumentando el consumo de energía, encarece la infraestructura y los servicios y tiene repercusiones negativas sobre la calidad del paisaje y el medio ambiente. Por otra parte, en algunas zonas, el aumento del nivel de vida ha incrementado la demanda de residencias secundarias, hasta el punto de que algunas aglomeraciones pueden considerarse como "ciudades de fin de semana".

Por otro lado, muchas veces el planteamiento de las ciudades no corresponde con las necesidades de los niños, es decir, para las familias con hijos pequeños, los barrios residenciales de la periferia suelen ofrecer una calidad de vida superior a la de los centros de las ciudades, y además, el deseo de poseer casa propia a menudo sólo puede materializarse allí, debido a la diferencia de precios. Por esta razón, numerosas familias con rentas medias o altas abandonan el centro de las ciudades en pro de las periferias.

Las medidas de desarrollo urbanístico han alterado a menudo el patrimonio histórico y el atractivo de las ciudades, así como su identidad. Ello tiene repercusiones negativas no sólo sobre la calidad de vida y la salud de sus habitantes, sino que también puede tener consecuencias económicas, debido a la pérdida de atractivo, al retroceso de las inversiones y del empleo, y a un debilitamiento de la situación financiera de las ciudades.

Por todas estas características la Estrategia Territorial Europea tiene un buen planteamiento a la hora de ayudar a las ciudades a planificar estrategias integrales que intenten coordinar las diferentes planificaciones sectoriales que puedan existir en una misma ciudad o entre ciudades cercanas y, poder crear así una red urbana equilibrada para el desarrollo del territorio. 


\section{LA FRONTERA ENTRE PAÍSES}

La idea de frontera, por un lado, sugiere las nociones de barrera y límite de división y discontinuidad, de divergencia de fuerzas centrífugas, por otro lado suscita, cada vez más, las nociones de permeabilidad y paso, de contacto de unión, de convergencia y cooperación.

La frontera, siendo una creación del espíritu y una construcción geopolítica, ha constituido un elemento importante en la organización y el desarrollo del territorio.

Los problemas de las regiones fronterizas españolas y portuguesas son de carácter estructural y revelan su posición periférica y marginal en el territorio y en los respectivos procesos nacionales de desarrollo. El territorio fronterizo entre España y Portugal se ve obstaculizado por su situación de doble periferia, atendiendo a su relación geo-económica en ambos contextos, el ibérico y el europeo.

El proceso de desarrollo rural al que han estado sujetas la mayoría de las regiones rayanas implica variables demográficas, económicas y sociales, creando innumerables obstáculos al deseado desarrollo sostenible. Este proceso de despoblamiento y desertificación, que afecta especialmente a la región extremeña, debilita la organización territorial y, al mismo tiempo, desvitaliza las pequeñas comunidades locales $\mathrm{y}$, a su vez, refuerza algunos crecimientos desiguales y polarizados, haciendo emerger a varios núcleos urbanos donde se concentra la mayor parte de la población.

Existen aún otros problemas o límites a la cooperación transfronteriza, concretamente, las diferencias entre los dos sistemas políticos, jurídicos y administrativos. Por ejemplo, en lo que respecta a España, existen Comunidades Autónomas y Juntas Provinciales; en Portugal no existe ningún interlocutor administrativo a nivel regional, siendo ese papel representado por los municipios. 
A pesar de las limitaciones y disfunciones que subsisten, la cooperación transfronteriza encierra virtudes que interesan al desarrollo de las regiones de frontera y que contribuyen a superar localismos y problemas comunes. En este sentido, se ha verificado, especialmente por parte de España, una fuerte voluntad de institucionalizar el diálogo entre las comunidades a ambos lados de la frontera, intensificando la cooperación entre agentes institucionales, económicos, sociales y culturales, de ámbito nacional, regional y local, para consolidar el deseado proceso de desarrollo de las áreas fronterizas y mejorar su inserción en contextos territoriales más importantes y globalizadores.

En las regiones de frontera la problemática de la cooperación transfronteriza tiene, necesariamente, que ser reordenada. La cooperación transfronteriza es, además, no sólo una vía para el desarrollo, sino una realidad. Basta pensar que España y Portugal poseen ríos y embalses internacionales y áreas protegidas que se prolongan a ambos lados de la frontera. En la perspectiva del Esquema de Desarrollo del Espacio Comunitario (EDEC)11 la posición fronteriza pasó a constituir un elemento potenciador del incremento de las relaciones entre territorios vecinos y, también, con otras regiones comunitarias y extracomunitarias.

\section{POLÍTICAS INTERNACIONALES Y DEMARCACIONES HÍDRICAS}

En las dos últimas décadas los temas relacionados con el agua han alcanzado una gran trascendencia política en los ámbitos nacionales e internacionales. Han sido numerosos los foros especialmente dedicados a los problemas hídricos y a las prolijas recomendaciones, directivas, leyes, reglamentos, etc. La Conferencia de Dublín en 1992 inaugura un camino que pasa por la Cumbre de la Tierra de Río de Janeiro (2002), ese mismo año, así como el II Foro Mundial del Agua de La Haya celebrado el año 2000 y la Conferencia de Bonn en 2001, la Cumbre de la Tierra de Johannesburgo (septiembre de 2002), la Semana Internacional del Agua (Estocolmo, 2008), 
continuando progresivamente en diferentes eventos internacionales, como se observa en el mapa 1, hasta concluir en Brasilia (2018)

Mapa 1. Conferencia internacionales del foro Mundial del agua

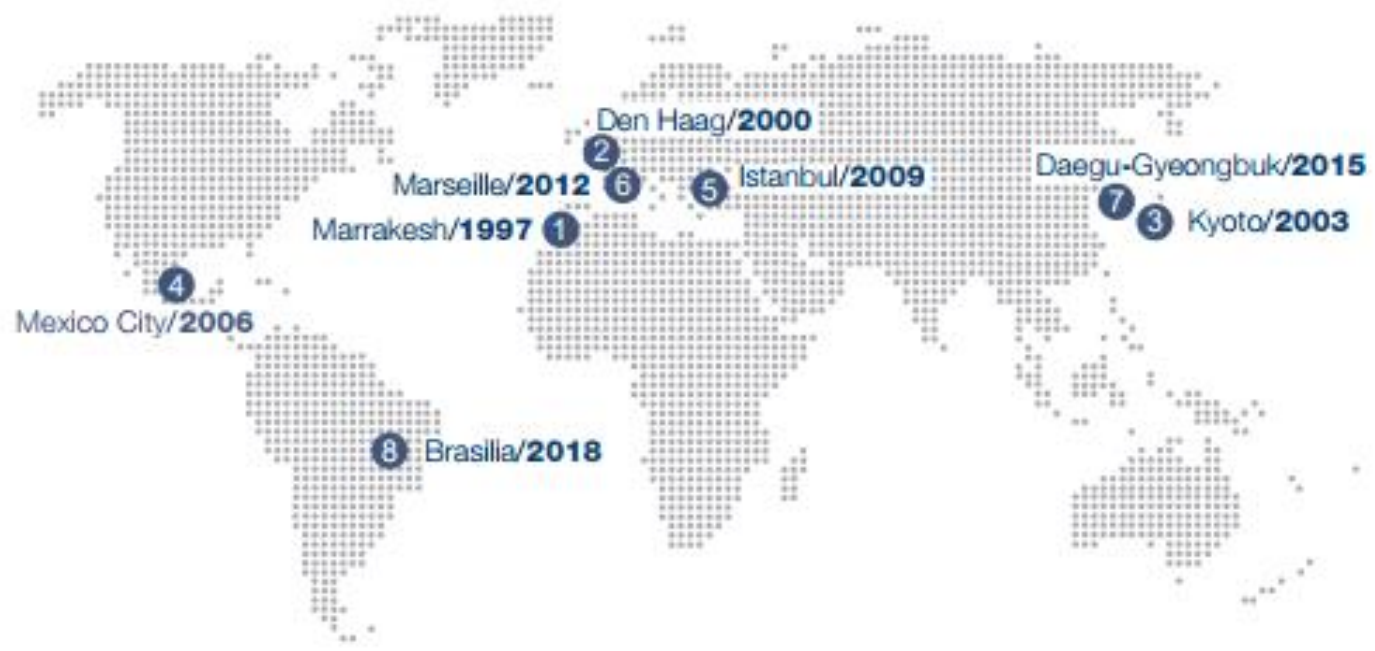

World Water Forum events since 1996

Fuente: http://www.worldwatercouncil.org/es/foro-mundial-del-agua

Como resultado de todo ello nuestro conocimiento de los tipos de cambio necesarios con respecto a la buena gestión y a los métodos para la ordenación de los recursos hídricos ha progresado considerablemente. Asimismo, está ampliamente aceptado que la gestión integrada de los recursos hídricos constituye la base teórica y el marco político para el uso sostenible y la preservación del agua y sus ecosistemas. Para hacer posible esa gestión integrada, sostenible y, en nuestro caso, compartida, resultan imprescindibles multitud de medidas de reforma, no sólo de las instituciones encargadas de la gestión del agua, sino también de los propios sistemas de análisis y evaluación de dicha gestión. 
A pesar de los indudables avances realizados hasta el momento, existen importantes obstáculos para la puesta en práctica de esta gestión integrada, compartida y sostenible de los recursos hídricos transnacionales: la fragmentación y superposición de los mandatos jurídicos e institucionales (Unión Europea, Estados diferentes -España y Portugal, Comunidades Autónomas y municipios), la resistencia a las reformas por parte de los numerosos actores involucrados, la falta de estructuras eficaces para fomentar una participación generalizada de los interesados y, sobre todo, la carencia de un sistema de indicadores y de evaluación de dicha gestión, que sea aceptado de mutuo acuerdo por las partes y que pueda constituir el punto de partida para cualquier debate político o técnico ulterior.

Hasta la fecha se han planteado distintos enfoques: en Dublín se formuló el reto de administrar los recursos hídricos teniendo en cuenta su valor en las dimensiones económica, social, ambiental y cultural. Este tema del valor de los recursos hídricos constituye, a nuestro modo de ver, uno de los pilares básicos de la gestión integrada, pero no es el único que se ha aplicado hasta la fecha. El análisis y evaluación de los modelos de gestión de agua se pueden plantear desde diversos ángulos, según el interés de las comunidades humanas:

Entre las diversas visiones que existen están las de un marcado carácter utilitarista del uso del agua, que abordan la gestión del agua fundamentalmente como elemento para el abastecimiento de la población; para la producción de alimentos; para uso energético; para uso industrial; o como mero medio utilitario

Otras visiones confieren a la gestión del agua un carácter más integrador dentro de los ámbitos de acción global. Son aquellas visiones de planificación integral de las relaciones con los otros factores vitales: el agua y las ciudades; el agua y la ordenación del territorio (la gestión del agua no puede producirse en abstracto, sino que debe relacionarse de manera estrecha con el resto de recursos naturales y ser un elemento básico para la ordenación del territorio, motivo por el cual las decisiones han de tomarse considerando las fronteras naturales, es decir a nivel de cuencas y 
demarcaciones hidrográficas); enfoque ecosistémico: cuando se pretende gestionar los recursos hídricos de forma sostenible se ha de considerar el posible impacto negativo de la actividad humana en el medio ambiente. Se debe aprender a respetar la base del recurso del cual la vida depende en última instancia y considerar la tierra, el aire y el agua como partes integrantes y de igual importancia de un mismo sistema; Usos recreativos, etc.

\section{PROPUESTAS METOLÓGICAS DE ACTUACIÓN EN LA FRONTERRA HISPANO- PORTUGUESA}

En este contexto constituye una condición necesaria el que la gestión integrada del agua se lleve a cabo en el marco de referencia de la Demarcación hidrográfica ${ }^{4}$, espacio definido en el que interactúan las actividades humanas con el medio físico. Al tratarse de un sistema geográfico, se superpone a las divisiones administrativas y políticas, resultando necesario definir un marco institucional específico que permita la integración de las actuaciones con la coordinación de los diferentes poderes públicos actuantes en el territorio, y que sirva para ser extrapolada esta experiencia como "Buena Práctica" internacional. Para ello, se debe evaluar la situación actual de la gestión de los recursos hídricos Hispano-Lusos

1. Diseñar para ello un sistema de evaluación basado en indicadores de resultado aplicados a la gestión integrada, compartida y sostenible del agua en dichas cuencas

\footnotetext{
${ }^{4}$ Demarcación hidrográfica: la zona marina y terrestre compuesta por una o varias cuencas hidrográficas vecinas y las aguas subterráneas y costeras asociadas, designada como principal unidad a efectos de la gestión de las cuencas hidrográfica (Directiva 2000/60/CE del Parlamento Europeo y del Consejo, de 23 de octubre de 2000; modificada por la Directiva 2008/32/CE del Parlamento Europeo y del Consejo, de 11 de marzo de 2008. Asimismo, en España, las demarcaciones hidrográficas vienen reguladas a través del Real Decreto 125/2007, de 2 de febrero, por el que se fija el ámbito territorial de las demarcaciones hidrográficas
} 
2. Seleccionar indicadores preexistentes que resulten de utilidad para dicha evaluación

3. Formular o desarrollar indicadores novedosos para aquéllos aspectos a evaluar que no cuenten con indicadores preexistentes

4. Divulgar, discutir y consensuar en foros científicos y técnicos Hispano-Lusos dicho sistema de evaluación e indicadores

Aplicar dicha metodología de evaluación a las cuatro demarcaciones hidrográficas transnacionales ibéricas: Miño-Lima, Duero, Tajo y Guadiana. Para cada una de las cuencas se pretende evaluar el carácter integrado, compartido y sostenible de la gestión del agua en materia de:

1. Satisfacción de las necesidades básicas hídricas

2. Suministro para producción de alimentos.

3. Preservación de la integridad de los ecosistemas.

4. Uso y gestión compartida de los recursos hídricos: marco normativo e institucional

5. Riesgos naturales y cambio climático: sequías, inundaciones e invasión de cauces.

6. Valorización del agua (económica, ambiental, social y culturalmente)

7. Mejora del conocimiento de base.

Definir propuestas de actuación que permitan mejorar el sistema de gestión de los recursos hídricos profundizando en el carácter integrado, compartido y sostenible del mismo, de modo que se pueda seguir avanzando en el cumplimiento de los objetivos antedichos mediante lo que sería, como en otras publicaciones hemos avanzado, una posible Estrategia Ibérica del Agua.

\section{BIBLIOGRAFÍA}


CONFERENCIA DE BONN (2001). Conferencia de Bonn sobre cambio climático. Disponible en:

COMISIÓN EUROPEA (1999). Estrategia Territorial Europea: Hacia un desarrollo equilibrado y sostenible del territorio de la UE. Disponible en:

. European Spatial Development Perspective: Towards Balanced and Sustainable Development of the Territory of the European Union. Disponible en:

Foro Mundial del Agua de La Haya (2000). Disponible en:

LÓPEZ, Lorenzo y PRIETO, Ignacio (1999). Evolución demográfica reciente y ordenación del territorio en Castilla y León. En la: Revista de investigación económica y social de Castilla y León, № 1, 87-102. Disponible en:

MORA Aliseda, J., GARRIDO Velarde, J. y DÍAZ González, M. (2016). Dinámicas socio-espaciales y previsiones demográficas en la cuenca Internacional del río Duero. Cuadernos Geográficos 55 (1), 221-238.

NACIONES UNIDAS (1992). Declaración de Dublín sobre el agua y el desarrollo sostenible. Conferencia Internacional sobre el agua y el medio ambiente. Disponible en:

. Declaración de Río sobre Medio Ambiente y el Desarrollo. Conferencia de las Naciones Unidas sobre el Medio Ambiente y el Desarrollo. Disponible en:

(2002). Cumbre de la Tierra de Johannesburgo. Conferencia de las Naciones Unidas sobre el Medio Ambiente y el Desarrollo. Disponible en:

Disponible en:

(2017). Decenio Internacional de Acción "Agua para la Vida" 2005-2015.

Los últimos acuerdos clave incluyen la Agenda 2030 para el Desarrollo Sostenible, el Marco de Sendái para la Reducción del Riesgo de Desastres 2015-2030, la Agenda de Acción de Addis Abeba 2015 sobre la Financiación para el Desarrollo, y el Acuerdo de París 2015 dentro del Marco de la Convención de las Naciones Unidas sobre el Cambio Climático.

WORLD WATHER WEEK. Compartiendo el Agua. Brasilia, 2008. 
Vertentes do Direito

WORLD WATHER WEEK. Progress and Prospects on Water: For a Clean and Healthy Word. Estocolmo, 2008. 